\section{New drugs for Alzheimer's disease}

\author{
ALISTAIR BURNS, EVE RUSSELL and SEAN PAGE
}

Alzheimer's disease is the most common cause of dementia in older people, affecting up to $10 \%$ of the population over the age of 65 years and three times that figure in those over the age of 90 years (Hofman et al, 1991), with a total of about half a million people affected in the UK. The cause of Alzheimer's disease is essentially unknown, there is no cure for the disease, the effect on sufferers and families is devastating in personal and emotional terms and the annual cost is nearly $£ 1$ billion in the UK (Bosanquet et al, 1998). Based on this background, there should be understandable enthusiasm at the introduction of new drugs for the treatment of the core features of the disease (the cognitive deficits, predominantly memory loss) based on amelioration of the underlying pathophysiology.

\section{BACKGROUND}

A new class of agents, the cholinesterase inhibitors (or anticholinesterases because of their inhibiting action on acetylcholinesterase, the enzyme responsible for the breakdown of acetylcholine), is now available for the symptomatic treatment of Alzheimer's disease. Other agents may influence disease progression, such as vitamin E (c.g. Sano et al, 1997), or are potentially active against both Alzheimer's disease and vascular dementia (e.g. propentofylline; Rother et al, 1998). The purpose of this article is to review briefly these new drugs and outline the implications for psychiatrists, particularly old age psychiatrists, upon whom the burden of care is predictably falling.

The clinical manifestations of dementia are threefold: cognitive impairment, noncognitive features (psychiatric symptoms and behavioural disturbances), and activities of daily living (ADLs). Measurement of the effects of drugs has to take into account the changes in these domains and their relative importance traditionally has determined which scales are used to assess drug efficacy. It is widely accepted that cognitive impairment is the primary abnormality in dementia but the clinical and practical relevance of a numerical improvement in a memory test has been questioned, leading to the introduction of global assessments, the most commonly used being the Clinician's Interview Based Impression (CIBI), which is a simple seven-point scale measure based on the assumption that a clinician can detect change in a patient's condition (Knopman et al, 1994, Schneider et al, 1997). Specific derivatives aimed at capturing change over time - the Clinicians's Interview Based Impression of Change (CIBIC; Leber, 1990) and a scale utilising care-giver information (CIBIC Plus; Leber, 1990)-are now accepted as being an essential component of any drug trial.

Non-cognitive features of dementia traditionally were regarded as epiphenomena (i.e. secondary to cognitive deficits), improvement in which would take place as a natural consequence of cognitive deficits and treatment of which was non-specific. For example, a positive effect by a drug on depressive symptoms would lead to the drug being regarded as an antidepressant and not an antidementia drug, even if a positive effect on cognition was demonstrated. Problems in ADL are categorised as basic (e.g. eating, toiletting, washing/ dressing) or instrumental (more complex, such as cooking, shopping and handling finances). The Committee for Proprietary Medicinal Products (CPMP, 1997) has produced guidelines recommending that two or three areas of dementia should be assessed to demonstrate efficacy - cognitive function and either a global rating or ADL.

\section{TETRAHYDROAMINOACRIDINE (TACRINE)}

Tetrahydroaminoacridine (tacrine) was the first of the new agents to be introduced and was licensed in the USA, Canada and parts of Europe in 1993. It improved cognitive function in up to $30 \%$ of people with Alzheimer's disease tested over a sixmonth period in doses of up to $160 \mathrm{mg}$ per day. Five crossover trials and five parallel group trials have been published on the efficacy of tacrine (Glennie, 1997) - modest efficacy overall and minor improvement in cognitive function were seen in only three. Peripheral cholinergic side-effects (such as nausea, vomiting and diarrhoea) were seen in one-fifth of patients but liver toxicity was more serious, with $40 \%$ of patients having raised hepatic transaminase levels (severe in $2 \%$ ) and necessitating blood monitoring of liver function tests for the first few months of treatment. In view of the high level of side-effects, tacrine cannot be recommended for routine use. The drug was not originally granted a licence in the UK but was approved at the same time as donepezil.

\section{DONEPEZIL HYDROCHLORIDE}

The development of a drug with a similar efficacy profile to tacrine but free of sideeffects was awaited and donepezil hydrochloride seemed to satisfy this criterion. As a piperidine-based anticholinesterase, it does have the same potential for liver toxicity as the acridine-based compound, tacrine. It was licensed for use in the symptomatic treatment of mild to moderate Alzheimer's disease in the UK on 17 March 1997. At the time of writing, there have been four published trials of donepezil - a Phase II randomised controlled trial (Rogers \& Friedhoff, 1996) with an openlabel extension (Rogers \& Friedhoff, 1998) and two double-blind Phase III placebo-controlled trials (Rogers et al, $1998 a, b)$ - all of which have taken place in the USA. A European and Canadianbased multi-country trial has been completed and the results are in the public domain. Donepezil was licensed at a stage when only the Phase II trial had appeared in a peer-reviewed journal, although data on the other trials were made available to the regulatory authorities. All three studies have demonstrated the benefits of donepezil compared to placebo. The Phase II study (Rogers \& Friedhoff, 1996) included 161 patients with mild to moderately severe Alzheimer's disease prescribed 1,3 or $5 \mathrm{mg}$ of active drug or placebo and found a statistically significant improvement in 
the ADAS-Cog score (cognitive sub-scale of the Alzheimer's Disease Assessment Scale; Rosen et al, 1984) in patients on 3 and $5 \mathrm{mg}$ compared with placebo. No difference was found with the other measures. Rogers et al (1998a) reported that cognitive function (ADAS-Cog), ADL and global functioning (CIBIC) improved in 473 patients taking 5 or $10 \mathrm{mg}$ compared with placebo, but with no difference between drug doses. The improvement returned to the level of the placebo group after a six-week washout. Rogers et al $(1998 b)$ reported a 12-week study on 468 patients and confirmed significant benefit of the drug over placebo; the Europeanand Canadian-based multi-centre trial has produced similar positive results (Burns $e t$ al, 1999). Side-effects with donepezil are generally of moderate severity, transient and consist of diarrhoea, vomiting, fatigue, muscle cramps and dizziness. They affect less than $10 \%$ of people and are higher with the $10 \mathrm{mg}$ dose.

The magnitude of change (similar in all studies) seen with donepezil is an improvement of around three points on active drug compared with placebo on the ADAS-Cog scale, which is highly statistically significant $(P<0.0001)$. To look at it another way, $42 \%$ of patients on placebo had worse cognitive function at the end-point compared with $20 \%$ on donepezil. An improvement of seven points on the ADAS-Cog scale is regarded as being of clinical significance; on this basis, $8 \%$ on placebo, $15 \%$ on $5 \mathrm{mg}$ and $25 \%$ on $10 \mathrm{mg}$ improved. On the CIBIC, $11 \%$ improved in the placebo group compared with $26 \%$ on $5 \mathrm{mg}$ and $25 \%$ on $10 \mathrm{mg}$. The recommended drug dose is to start with $5 \mathrm{mg}$ per day and titrate after a minimum of one month to $10 \mathrm{mg}$. In the UK, the cost of the drug is $£ 68.32$ for $285 \mathrm{mg}$ tablets and $£ 95.76$ for $2810 \mathrm{mg}$ tablets.

\section{RIVASTIGMINE}

Rivastigmine was licensed on 12 May 1998 in the European Community for the treatment of mild to moderately severe Alzheimer's disease. Compared with donepezil, there is a larger patient database on which the introduction of the drug was based. A specific programme of studies (the ADENA programme; Anand \& Gharabani, 1996; Novartis website (http:// www.alzheimer-info.com/exelon), 1998) consisted of four pivotal trials aimed at overcoming the drawbacks of existing studies (i.e. small numbers of patients, short duration of treatment and restrictive entry criteria). Over 2000 patients have been examined with a dose range of 6-12 mg (twice daily administration), previous studies having shown that $4 \mathrm{mg}$ was ineffective. The magnitude of the effect is similar to that seen with donepezil. Specific measures of ADL were included in the trials (the Progressive Deterioration Scale (PDS); de Jong et al, 1989). Compared with placebo, 21 v. $12 \%$ improved on ADAS-Cog, 29 v. $18 \%$ improved on the CIBIC Plus and $26 v .17 \%$ improved on the PDS. Symptoms on rivastigmine included anorexia, nausea, dizziness, vomiting, appetite and weight loss. It is not, like donepezil, contraindicated in asthma. The recommended drug dose is to start at $1.5 \mathrm{mg}$ twice daily with the therapeutic dose between 6 and $12 \mathrm{mg}$. In the UK, the cost of the drug is $£ 31.50$ for 28 tablets regardless of size $(1.5,3,4.5$ or $6 \mathrm{mg})$, giving a monthly price of $£ 63$ for $12 \mathrm{mg}$ per day.

\section{OTHER DRUGS}

A dose-finding study of metrifonate (Morris et al, 1998) demonstrated the efficacy and safety of the drug in patients with Alzheimer's disease, showing improvements in ADAS-Cog and the CIBIC. Morris et al (1998) described a 26-week double-blind treatment trial followed by an 8-week placebo extension on 408 patients with Alzheimer's disease, of whom over $80 \%$ completed the trial in the active treatment group. A highly significant improvement was found in the ADAS-Cog on an intention -to-treat basis. In addition, a specific beneficial effect was found in terms of noncognitive features as measured by the Neuropsychiatric Inventory (NPI; Cummings et al, 1994). The beneficial effect of the drug on non-cognitive features of dementia is novel. McKeith (1998) described an international study with 605 patients over 26 weeks in doses of $40-80 \mathrm{mg}$ per day. On an intention-to-treat basis, the drug showed clear benefit over placebo on cognitive performance and global function. There are currently safety concerns about metrifonate.

Other anticholinesterase agents are being tested (such as galanthamine and slow-release physotigmine, eptastigmine, ibedenone, zifrosilone and quilostigmine), as are cholinergic muscarinic agonists (e.g. melamiline and xanomeline) and precursor loading agents (acetyl- $l$-cartinine). Noncholinergic treatments include: propentofylline, an endenozyne reuptake (phosphodiesterase) inhibitor that, it is claimed, modulates mechanisms in Alzheimer's disease and vascular dementia - cell activation, increased production of cytokines, free radicals and glutamate - with benefits seen in 901 patients with Alzheimer's disease and 359 patients with vascular dementia (Rother et al, 1998); memantine, a modulator of the glutamatergic system that modulates the cytotoxic effects of excitatory amino acids and has been shown to be of benefit in both Alzheimer's disease and vascular dementia (Gortelmeyer et al, 1993); vitamin E and selegiline (Sano et al, 1997), alone and in combination, which delay the progression of Alzheimer's disease; Ginkgo biloba (Le Bars et al, 1997), which has been shown to produce benefit in one study; Cox-2 inhibitors (drugs with an anti-inflammatory action), which are being tested; and calcium channel blockers (e.g. sabeluzole and nimodipine), which have been used. Oestrogens also are being tested as potential agents for the prevention of Alzheimer's disease (Burns \& Murphy, 1996).

\section{MANAGED INTRODUCTION - CLINICAL GUIDELINES}

Guidelines have been used to manage the introduction of the new drugs for the treatment of Alzheimer's disease (e.g. Lovestone et al, 1997). They define diagnostic criteria for Alzheimer's disease, suggest clinically relevant inclusion and exclusion criteria based broadly on those used in the clinical trials and, ideally, should be agreed locally with general practitioners, pharmacists, local purchasers of health care and specialists in the management of Alzheimer's disease. As with many other drugs, there is significant variation between different areas of the country and the implementation of guidelines (Harvey, 1999). Many purchasers of health care remain to be convinced of the usefulness of prescribing these drugs in dementia, and the tone of the two articles in the influential Drugs and Therapeutics Bulletin review of donepezil reflects this attitude (Anonymous, 1998, 1999).

The UK Government's Standing Medical Advisory Committee (SMAC, 1998) has produced guidelines on the treatment of Alzheimer's disease and recommends that treatment should be initiated and 
supervised by a specialist familiar with the management of Alzheimer's disease and that, generally speaking, the characteristics of patients selected for treatment should be the same as those in drug trials. Treatment should be assessed after 12 weeks and continued only if there is clear evidence of benefit, a judgement informed by the application of recognised objective tests.

\section{CLINICAL EXPERIENCE}

Our clinical experience in south Manchester is that the drugs were introduced without additional funding using a locally agreed protocol formulated by primary care, hospital specialists and pharmacists. The criteria are similar to those described in the SMAC guidelines. Prescribing is initiated by consultants in old age psychiatry in the setting of a specialist multi-disciplinary domiciliary clinic. Treatment is targeted towards patients with mild to moderate Alzheimer's disease supported by radiological evidence of the absence of vascular disease. Measurements of efficacy in the three domains of cognitive function, ADL and non-cognitive features of Alzheimer's disease are made before the start of treatment and at three-monthly intervals thereafter.

Early experience of donepezil is similar to that of the drug trials in respect of the change in cognitive function, with about half of patients showing improvement at six months and two-thirds of the remainder failing to show the expected deterioration. Of more clinical importance are the striking improvements in non-cognitive features and ADL in some patients. Moreover, their carers describe marked changes to their own quality of life by no longer having to cope, for example, with a spouse or parent hallucinating or wandering at night. Many carers remark that patients appear brighter and more interested in their surroundings. The cost benefit of such changes has been questioned but the benefit to patients and carers is clear to them.

As might be expected, when the drug is introduced into clinical practice with patients experiencing medical comorbidity, the adverse effect profile is worse than that seen in the trial data. Our initial protocol titrated all patients on donepezil up to $10 \mathrm{mg}$ after a month and, perhaps as a result of this relatively quick titration (albeit that given in the data sheet), the frequency of adverse effects was high. Patients now

ALISTAIR BURNS, FRCPsych, EVE RUSSELL, MRCPysch, SEAN PAGE. MHSM, Department of Psychiatry. University of Manchester. Withington Hospital, Manchester

Correspondence: Professor A. Burns. Department of Psychiatry. University of Manchester. Withington Hospital. Manchester M20 8LR, UK. Tel: 0161291 3310; Fax: 01612914364

(First received 16 November 1998, final revision 3 February 1999, accepted 2 March 1999)

receive $5 \mathrm{mg}$ and side-effects are usually mild and self-limiting.

Rivastigmine has been used less often but, as with donepezil, we have seen striking effects on non-cognitive features. Patients who have failed to tolerate donepezil appear to tolerate rivastigmine at a therapeutic dose and medical comorbidity seems to be less of a problem than with donepezil, particularly in relation to chronic obstructive airways disease and asthma. However, the twice daily dosage is a problem for patients without a carer, with whom we struggle to ensure compliance even on once daily donepezil.

Overall, experience of these drugs has been very positive. Sixty-two patients have been started on treatment (3:1 in favour of donepezil) and approximately one-third (for each drug) have stopped treatment because of side-effects, intercurrent illness or lack of benefit. The changes seen in the ratings parallel those reported in clinical trials, although responses vary greatly between patients (Dening \& Lawton, 1998). Early and frank discussion at the start of treatment with patients and carers, with an agreed trial of treatment and discussion of the results, has ensured that we have experienced no problems with stopping the drugs if patients are not receiving any benefit from them.

\section{THE FUTURE}

New drugs for Alzheimer's disease are not cures - their effects are probably best described as modest. However, for the first time, symptomatic improvement in cognitive function in Alzheimer's disease is possible. Fear of the cost involved was calculated speedily in people's minds simply by multiplying the numbers of patients with dementia in the UK (800 000) by the cost of the drug per year $(£ 1000)$; this prompted a knee-jerk reaction against these drugs, masquerading as protection of public funds in the face of minimal clinical improvement. More pragmatic trials are needed, emphasised by the recent call for proposals from the Health Technology Assessment programme. The AD2000 trial in the West Midlands is currently underway. In practice, not everyone with dementia has Alzheimer's disease, not everyone with Alzheimer's disease has mild to moderate disease and even then not everyone will be suitable. Patients and their families may not even want the drugs. Improvements in patients may cause problems of their own, such as the desire to drive again as a result of improved memory and concentration. All the thoughtful clinician would ask for would be that the medication be available and be introduced in a responsible fashion using agreed clinical guidelines and subject to audit. One would seek parity with the controls and restrictions placed on other newly introduced agents for other conditions. The cost of the drugs may be high, but the human cost of dementia is infinitely higher.

\section{REFERENCES}

Anand, R. \& Gharabini, G. (1996) Clinical development of Exelon. The ADENA programme. Journal of Drug Development and Clinical Proctice. 8 $117-122$

Anonymous (1998) Donepezil update. Drugs and Theropeutics Bulletin, 36, 60-61.

- (1999) Donepezil for Alzheimer's disease. Drugs and Theropeutics Bulletin, 35, 75-76

Bosanquet, N., May, J. \& Johnson, N. (1998) Alzheimer's Diseose in the United Kingdorn. Burden of Diseose and Future Core. Health Policy Review Paper No. 12. London: Health Policy Unit, Imperial College School of Medicine

Burns, A. \& Murphy, D. (1996) Protection against Alzheimer's disease Loncet, 348, 420-421.

_, Rossor, M., Hecker, J., et al (1999) The effects of donezepil in Alzheimer's disease - results from a multinational trial. Dementia and Geriotric Cognitive Disorders, 10. 237-244.

Committee for Proprietary Medicinal Products (CPMP) (1997) Note for Guidance on Medicinal Products in the Treatment of Alzheimer's Disease. London: European Agency for the Evaluation of Medicinal Products.

Cummings, J., Megam, M. \& Gray, K. (1994) The Neuropsychiatric Inventory. Neurology, 44. 2308-2314. 
de Jong, R., Ostertund, O. \& Roy, G. (1969)

Measurement of quality of life changes in patients with Alzheimer's disease. Clinical Theropeutics, II, 545-555.

Denine, T. Lewton, C. (1998) Doctors want to offer more than sympathy. British Medical journal, 317. 945.

Gbonnie, G. (1997) The efficacy of tacrine and the measurement of outcomes in Alzheimer's disease. In Technology Overview: Pharmoceuticals, issue 5. Ottowa: Canadian Co-ordinating Office for Health Technology Assessment.

Cortedmopor, R., Kante, M., Parsons, C., et of (1993)

The treatment of dementia syndrome with ecotonal memantine. In Spectrum KTRUM der Negro-rehabilitation (ed.W.Von Wild), pp. 50-56. Munich: Zuckschwerdt.

Harvey, R. (1999) A review and commentary on a sample of 15 UK guidelines for the drug treatment of Alzheimer's disease. International journal of Geriatric Pychioury, in press.

Hofiman, A., Rocca, W., Brayne, C., ef of (1991) The prevalence of dementia in Europe. International Journal of Epidemiology, 20. 736-748.

Knopman, D, Krapp, M., Grecon, S., et al (1994) The Clinician Interview Based Impression (CIBI). Neurology, 44, 2315-2321.

Le Bars, P., Katz, M., Berman, N., ef al (1997) A placebo controlled double blind randomised trial of an extract of Ginkgo bilobo for dementia. Journal of the Americon Medical Association, 278, 1327-1332.

Leber, P. (1990) Guidelines for the Clinical Evoluation of Anti-Dementio Drugs. Open Letter to the Phormoceutical Industry Washington, DC: Food and Drug Administration.

Lovestone, S., Graham, N. \& Howard, R. (1997) Guidelines on drug treatment for Alzheimer's disease. Lancet. 350. 232-233.

Mckeith, 1. (1988) The clinical trial protocol of Metrifonate in Alzheimer's Trial (MALT). Dementio \& Geriatric Cognitive Disorders, 9 (suppl. 2), 2-7.

Morris, J., Orrus, R, Oraxem, J., et of (1998) Metrifonate benefits cognitive, behavioural and globa function in patients with Alzheimer's disease. Neurology 50. $1222-1230$

Rogers, S. \& Friedhof, L. (1996) The efficacy and safety of donepezil in patients with Alzheimer's disease. Dementio, 7. 293-303.

_ _ (1990) Long-term eff icacy and safety of donepezil and the treatment of Alzheimer's disease. European Neuropsychophormocology, 8 $67-75$.

_, Farlow, M., Doody, R., ef al (Is9sa)

A 24-week double-blind placebo-controlled trial of donepezil in patients with Alzheimer's disease. Neurology, 50, 136-145.
Doody, R., Mohs, R., ex of (1992b)

Donepezil improves cognition and global function in Alzheimer's disease. Archives of Internal Medicine, 158, $1021-1031$.

Rosen, W, Mohs, R. Davles, K. (1984) A new rating scale for Alzheimer's disease. Americon fournol of Psychiotry, 141, 1356-1364.

Rother, M., Erkinjunti, T., Roessner, M., et al (1998) Propentofylline in the treatment of Alzheimer's disease and vascular dementia. A review of Phase 3 trials. Dementio \& Geriatric Cognitive Disonders, 9 (suppl. 1), 36-43.

Sano, M. Ernesto, C., Thomas, R., ot al (1997) A controlled trial of selegiline, alpha tocopherd or both as treatment for Alzheimer's disease. New England fournol of Medicine, 336. 1216-1222

Schnolder, L., Olin, J., Doody, R., of of (1997) Validity and reliability of the Alzheimer's Disease Co-Operative Study - clinical global impression of change. Alzheimer's Diseose and Associoted Disorders, II (suppl. 2), \$22-\$32.

Standing Medical Advisory Committee (SMAC) (1998) The Use of Donepezil for Azheimer's Disease. London: Department of Health. 